\title{
Developments of Yoctowell Cavities as a Nano and Bio-technological Tool
}

\author{
Anushri Rananaware ${ }^{1}$, Melissa M A Kelson ${ }^{1}$, Hemlata Patil ${ }^{1}$, Aaron M Raynor ${ }^{1}$, Duong DucLa ${ }^{1}$, \\ Sam Leslie Jackson ${ }^{1}$ and Sidhanath V Bhosale ${ }^{2}$ and Sheshanath V Bhosale ${ }^{1 *}$ \\ ${ }^{1}$ School of Applied Sciences, RMIT University, Melbourne, Victoria, Australia \\ ${ }^{2}$ Polymers and Functional Material Division, Indian Institute of Chemical Technology, Hyderabad, Telangana, India
}

Received: July 31, 2014; Accepted: October 20, 2014; Published: October 30, 2014

*Corresponding author: Sheshanath V. Bhosale, School of Applied Sciences, RMIT University, Melbourne, 3001, Australia, Tel: +61-(03)99252680; E-mail: sheshanath.bhosale@rmit.edu.au

\section{To the Editor}

Biological energy conversion and molecular recognition processes normally occur on a nanoscale level. The PRCs (photosynthetic reaction centres) is one of the best example of energy conversion, which are equipped with protein helices in combination with ATP, porphyrins, and co-enzymes; such machinery of life was developed biologically in a long-term evolutionary process on a 1-10 yoctoliter $\left(1 \mathrm{yL}=10^{-24} \mathrm{~L}\right.$ or $\left.1 \mathrm{~nm}^{3}\right)$ scale [1]. So far, scientists have been successful with the isolation and analysis of such systems but not with reproduction of the working molecular apparatus. We have introduced a simple system of so-called "yoctowells", rigid molecular monolayers made of $\alpha, \omega$-diamido bolaamphiphiles around a porphyrin island of yoctoliter-sized volume, on to gold electrodes, colloidal gold particles, silica particles and magnetic silica particles (Figure 1) $[1,2]$.

The main motivation to work with membrane gaps, such as yoctowell systems, instead of proteins is their simplicity it would make it simpler to answer biological questions, which are very difficult to approach in complex biological systems. Designer yoctowells which may act as nano- and bio-technological tools via manipulating the interactions between guest molecules and the walls of the yoctowell gap were thought to offer an opportunity for studying intermolecular interactions [3]. The inner properties of the yoctowell can be fine-tuned by the nature of the bolaamphiphile monolayers (hydrophobic, hydrophilic and peptidic) to exploit the resulting electrostatic effects, which can be used to discriminate between substrates. The importance of these yoctowell cavities is the use of porphyrins as an optical sensor by means of absorption and emission in the host-guest event. Herein, we give a brief description of the developments of yoctowells and their usefulness in various technological applications. The stability of yoctowells can be confirmed by size exclusion fluorescence quenching experiments. Large molecules over $20 \AA$ in diameter cannot enter into the gaps whereas molecules with size proportionate with that of the yoctowell gaps are able to enter and quench the fluorescence of the base porphyrin, whose role is both as a structural element and as a fluorescence reporter. In 2000, fluid and rigid yoctowells had been developed and used as carriers for heterodimers of porphyrins on gold platelets and colloidal gold nanoparticles [4-8]. However, one drawback of gold-based systems is plasmon absorption, which can cause problems (quenching the florescence of the base porphyrin) in photophysical studies.

We then prepared yoctowell systems on photoinactive, amino-coated, silica particles. These particles are colorless, keeping the porphyrin fluorescence intact, and can be prepared using a variety of conditions with different coatings [9]. We have optimised these conditions and prepared particles with a perfectly smooth surface and uniform diameter of $100 \mathrm{~nm}$. The rigid yoctowells were prepared in two steps via self-assembly; first, covalently attachment of activated tetra-carboxy porphyrins to amino functional silica particles followed by binding of diamidobolaamphiphiles. The two amide bonds of the bolaamphiphiles rigidify the walls of the yoctowells. Furthermore, the double bonds of the bolaamphiphiles (walls) of yoctowells were functionalized at the double bond by the Michael addition of methylamine for the electrostatic interactions with anionic porphyrin. The ring of methylammonium groups was then fixed to the walls of the yoctowells at a distance of 5 or $10 \AA$ with respect to the base porphyrin and the precisely fitting manganese(III)meso(tetraphenyl-4-sulfonato) porphyrins (Mn(III)TPPS) was tightly bound to the ammonium groups. Thus, two different dyes (base porphyrin 1 and Mn(III)TPPS 3) have thus been immobilized at a defined subnanometer distance in an aqueous medium (Figure 2). Transient fluorescence experiments have shown a fast decay time of $0.2 \mathrm{~ns}$ for the porphyrin at the base of the yoctowell when the Mn(III)TPPS was fixed at a distance of $5 \AA$.

Importantly, hydrophobic yoctowells are also stable in organic solvents, such as ethanol and chloroform, so that when the second porphyrin was added (in bulk), it can enter into the wells freely and become irreversible fixated within the well [10]. Although, once it reach the base of the yoctowells they stacks irreversibly, but migration of these fitting porphyrins occurs extremely slowly. 


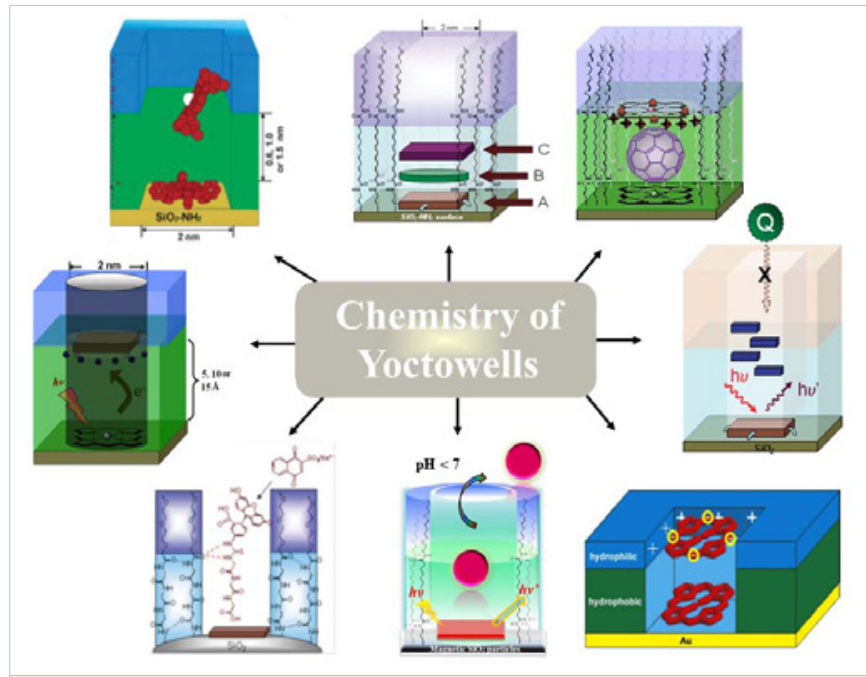

Figure 1: Schematic Illustration of the Yoctowell Cavities.

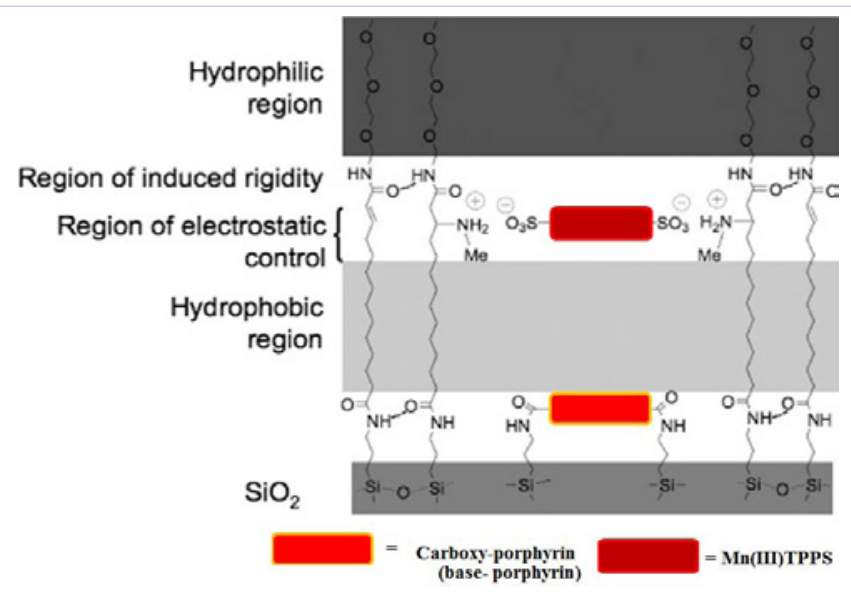

Figure 2: Representation of the yoctowell cavities on the silica surface and their functionalization with Michael addition with methaylammonium, followed by capping by the charged Mn(III)TPPS porphyrin 2 .

Taking advantage of the slow and irreversible adsorption process, a "sorting" of noncovalent porphyrin stacks in the order $\mathrm{A}, \mathrm{B}, \mathrm{C}$ and $\mathrm{A}, \mathrm{C}, \mathrm{B}$ (Figure 1) within the yoctowells in any order has been established for the first time. The molecular sorting was characterized by UV-vis, sequence dependent fluorescence quenching, and cyclic voltammetry of the porphyrin capping the yoctowells. Hydrophobic yoctowells have also been used to study kinetic trapping of molecules within the cavity [11]. Rigid-edged amphiphiles migrate from the bulk solution into the yoctowells and render them inaccessible to water-soluble ions. This blocking effect of the solutes is stereoselective. Trans-1,2-cyclohexanediol or cellobiose with equatorial hydroxyl-groups are very efficient blockers, whereas cis-1,2-cyclohexanediol or maltose with axial substituents are ineffective.

To further development, we replaced the hydrophobic walls with functional hydrophilic (tetraethyleneglycol) and peptidic (diglycinyl triamide) walls [12]. The hydrophilic yoctowells have been to study the binding of oligoamines (spermine, polylysine
$(M w t=300,000)$, and the rigid tricyclic tetraamine tobramycin in water at $\mathrm{pH}$ 7-8. The results show the binding equilibrium constant $(K)$ of spermin to be $\sim 10^{3} . \mathrm{M}^{-1}$, whereas polylysine and tobramycin block the yoctowells with $K=10^{7} \mathrm{M}^{-1}$. This kinetic trapping is much higher than binding of these guests in 3D crown ethers [13]. Peptidic yoctowells bind triglycine derivatives of fluorescein in $2: 1$ ratio with a $K>10^{13} . \mathrm{M}^{-1}$ which enables the number of entrapped molecules in each wells to be estimated by fluorescence comparisons.

Hydrophobic zinc-porphyrin-based yoctowells have also been used as a receptor for the formation of stable inclusion complexes of fullerenes via p-electron donor-acceptor (base porphyrin- $\mathrm{C}_{60}$ ) and hydrophobic interactions. Methylammonium rings in the walls are used to attach Mn(III)TPPS (an anionic porphyrin) to cover the wells [14]. This is the first example where pores have been used for the sequential one- dimensional stacking of molecules. Such superstructures consisting of photoactive dyes may be useful for studying electron-transfer phenomena.

Having hydrophobic and hydrophilic yoctowells in hand, we studied the binding discrimination of catecholamines (neurotransmitters), namely dopamine, adrenaline, noradrenaline, tyramine and serotonin, in aqueous solution by means of fluorescence quenching and cyclic voltammetry (CV) experiments [15]. The results clearly demonstrate that hydrophobic yoctowells are efficient receptors for dopamine, noradrenaline, adrenaline and tyramine and act by a process of nanocrystallization. However, serotonin which is indole based, possess no blocking effect. On other hand, hydrophilic yoctowells with differing magnitudes inferring that discrimination is likely on a competitive basis i.e. we only observe less than $10 \%$ blockages with all the catecholamines used in this study.

Bacterial PRCs are capable of light-induced microsecond charge-separation processes and use several membrane proteins to fixate the functional components with precise distances and orientations. To understand this phenomenon, we prepared three yoctowells ( $20 \AA$ in diameter) with varying depths of 5, 10, and $15 \AA$ measure from the positively charged rim; these gaps were prepared through a Michael addition of the methylammonium group right below the oligoethylene chain. Electrostatic interactions between the positively charged rim and anionic quinones enabled a base porphyrin-quinone (donor-acceptor) dyad to be prepared. Upon photoexcitation of the base porphyrin, an efficient distance-dependent electron transfer to the quinone unit takes place on the nanosecond time scale [16].

The non-swelling behaviour of the walls of the yoctowell also allow for dissolution of molecules in a range of solvents. The most characteristic property of yoctowells is their ability to induce the formation of well-filling "nanocrystals" in dilute $[0.1$ M) aqueous solutions using cyclic and rigid edge amphiphiles and to allow molecular sorting. Interestingly, hydrophobic yoctowells can be functionalized at molecular level in order to control the encapsulation and release of bioactive molecules for drug-delivery systems [15]. Recently, we demonstrated the use of yoctoliter wells as a simple model system for the encapsulation and release of biologically active molecules, facilitated by 
manipulating the pH. Doxorubicin (DOX; cancer drug) was encapsulated into the wells and stabilized by hydrophobic and Vander Waals interactions; the wells were capped by Mn(III) TPPS, which were held in place by electrostatic interactions [17]. The encapsulated DOX and Mn(III)TPPS guest molecules could be released from the wels walls by altering the $\mathrm{pH}$ of the solution. At lower pH (7-3.0) only Mn(III)TPPS was released, whereas at $\mathrm{pH}$ 7.2-9.4, the slow release of DOX was observed. Thus, both molecules were released from the wells by manipulating naturally occurring stimuli in vivo, i.e. $\mathrm{pH}$.

Yoctowell system on aminated-silica particles were shown to be very effective controlling the encapsulation and release of bioactive molecules, these drug-delivery yoctowell systems relied on non-active silica nanoparticles and had limitations for effective targeted drug delivery [17]. To overcome this problem, very recently, we have improved the existing yoctowell system by incorporating magnetic nanoparticles for the controlled release of the loaded drug to target areas [18]. Yoctowell cavities on magnetic silica nanoparticles were used for the encapsulation of the drug molecule mitoxantrone (MTZ), and controlled release could be achieved by altering naturally occurring stimuli, that is, pH. Firstly, MTZ was encapsulated from a bulk solution under physiological conditions and then released from the yoctowells in a controlled manner by manipulating the $\mathrm{pH}$ to $7.0-3.0$. The sustained release of MTZ, the recovery of active yoctowells after the release process and the magnetic properties of nanoparticles may serve as a foundation for the further development of a new generation of drug-delivery systems.

In summary, yoctowell systems with various kinds of walls can be precisely constructed through a range of techniques and are dependent on the particle surface employed. The integrity of the yoctowells can be confirmed by size-exclusion fluorescence quenching experiments. Yoctowells with hydrophobic and hydrophilic side walls are easy to prepare and characterize which may be used as simple models for biological systems. The most characteristic properties of the yoctowell are their ability to induce the formation of well-filling 'nanocrystals' in dilute $(0.1$ $\mathrm{M})$ aqueous solutions using cyclic and rigid edge amphiphiles, such as cellobiose, tyrosine or drug candidates, as well as their ability to allow molecular sorting to be studied. The non-swelling behaviour of the walls of the yoctowell allow for dissolution of molecules in a range of organic and aqueous solutions. Modification of the walls has enabled a strong design element for studying inclusion phenomena, typically with high association constants. Future directions in the applications of yoctowell models may include investigations of the recognition processes between steroid hormones and branched oligo-glycosides. Further studies on distant dependent electron transfer processes and in applications of $\mathrm{pH}$ dependent release of bioactive molecules in vivo applications. Ultimately, the versatility of the yoctowell system and its applications in the areas of nano- and bio-science for medicinal applications shows great promise.

\section{Acknowledgement}

SVB acknowledges, The Australian Research Council for providing financially support under a Future Fellowship Scheme (FT110100152). We thank Dr. Steven Priver for corrections and suggestions.

\section{References}

1. Mauzerall D, Hong FT, (1975) Porphyrins and metalloporphyrins, photochemistry of porphyrins in membranes and photosynthesis (Ed.: K. Smith), Elsevier, Amsterdam, The Netherlands, p. $707 \mathrm{f}$.

2. Bhosale SV, Langford SJ. Recent development of the yoctowells for investigation in nanospace. Chem Soc Rev. 2012; 41(5):1637-1651. doi: $10.1039 / \mathrm{c} 1 \mathrm{cs} 15152 \mathrm{e}$.

3. Wang T, Bhosale S, Bhosale S, Li G, Fuhrhop JH. Hydrophobic and hydrophilic yoctowells. Acc Chem Res. 2006; 39(8):498-508.

4. Bhosale SV, Langford SJ. The development of yoctowells as a basis for modeling biological systems. Org Biomol Chem. 2007; 5(23):37333744.

5. Li G, Fudickar W, Skupin M, Klyszcz A, Draeger C, Lauer M, et al. Rigid lipid membranes and nanometer clefts: motifs for the creation of molecular landscapes. Angew Chem Int Ed Engl. 2002; 41(11):18281852.

6. Skupin M, Li G, Fudickar W, Zimmermann J, Röder B, Fuhrhop JH. Methylammonium groups at the solid walls of nanometer-sized, water-filled monolayer gaps as binding sites for a tetraanionic porphyrin. J Am Chem Soc. 2001;123(15):3454-3461.

7. Fudickar W, Zimmermann J, Ruhlmann L, Schneider J, Röder B, Siggel $\mathrm{U}$, et al. Fluorescence quenching and size selective heterodimerization of a porphyrin adsorbed to gold and embedded in rigid membrane gaps. J Am Chem Soc. 1999;121(41):9539-9545. doi: 10.1021/ ja991738n.

8. Li G, Fuhrhop JH. Anticorrosive lipid monolayers with rigid walls around porphyrin-based $2 \mathrm{~nm}$ gaps on $20 \mathrm{~nm}$ gold particles. Langmuir 2002; 18(20):7740-7747. doi: 10.1021/la020526s.

9. Li G, Bhosale SV, Wang T, Hackbarth S, Roeder B, Siggel U, et al. Nanowells on silica particles in water containing long-distance porphyrin heterodimers. J Am Chem Soc. 2003; 125(35):1069310702.

10. Bhosale S, Bhosale S, Wang T, Li G, Siggel U, Fuhrhop JH. Slow motion, trapping and sorting of water- and chloroformsoluble porphyrins in nanowells. J Am Chem Soc. 2004; 126(40):13111-13118.

11. Bhosale S, Li G, Li F, Wang T, Ludwig R, Emmler, T et al. Counting of labeled tyrosine molecules in hydrophobic yoctolitre wells filled with water. Chem Commun (Camb). 2005; (28):3559-3561.

12. Bhosale S, Bhosale S, Wang T, Kopaczynska M, Fuhrhop JH. Hydrophilic and hydrophobic yoctowell as a receptor for single, water-soluble molecules. J Am Chem Soc. 2006; 128(7):2156-2157. doi: 10.1021/ ja053816e.

13. Cram DJ, Cram JM, (1994) In container molecules and their guests; Stoddart, J. F., Ed.; RSC: Cambridge.

14. Bhosale SV, Bhosale SV, Langford SJ, Krsta D. Construction of trimeric porphyrin-fullerene-porphyrin stacks within surface-derived pores of nanoscale dimensions. Chem Commun (Camb). 2009; (22):3166-8. doi: 10.1039/b903699g.

15. Bhosale SV, Langford SJ, Bhosale SV. Comparative binding study of neurotransmitters in hydrophobic and hydrophilic yoctowells in water. Supramol Chem. 2009; (21):18-23. doi: 10.1080/10610270802438838. 
16. Bhosale SV, Hackbarth S, Langford SJ, Bhosale SV. Light-induced electron transfer over distances of 5,10 and $15 \AA$ within waterfilled yoctowells. Chem Asian J. 2012; 7(1):176-182. doi: 10.1002/ asia.201100533.

17. Bhosale SV, Bhosale SV. Yoctowells as a simple model system for the encapsulation and controlled release of bioactive molecules. Sci Rep. 2013; 3:1982. doi: 10.1038/srep01982.

18. Bhosale SV. Yoctowell Cavities on Magnetic Silica Nanoparticles for $\mathrm{pH}$ Stimuli-Responsive Controlled Release of Drug Molecules. Chemistry. 2014; 20(18):5253-5257. doi: 10.1002/chem.201400279. 